\title{
A CCD SURVEY OF BL LAC OBJECTS
}

\author{
Helmut Kuhr and Josef Fried \\ Max-Planck-Institut fur Radioastronomie \\ Heidelberg, F.R.G.
}

\begin{abstract}
A long term project was started at the Max-Planck-Institut fur Astronomie, Heidelberg, to study a complete sample of 46 northern and southern BL Lac objects with flux densities exceeding 1 Jy at $5 \mathrm{GHz}$ using optical spectroscopy, optical polarimetry, and direct deep CCD imaging.
\end{abstract}

\section{INTRODUCTION}

Several studies have been published where authors discuss the underlying galaxy content of quasars (e.g. Gehren et al. 1984). Little is known, however, about the nature and morphology of the galaxies BL Lac objects are possibly imbedded in. The subject of this presentation is an optical direct imaging program concentrating on a complete sample of 46 radio selected BL Lac objects.

\section{THE SAMPLE}

The complete sample contains all objects from the $1 \mathrm{Jy}$ catalogue (Kuhr et al. 1981) covering the northern and southern sky, which meet the following criteria :

i) at the time of the radio surveys flux densities exceeded $1 \mathrm{Jy}$ at 5 $\mathrm{GHz}$, ii) on Palomar Observatory Sky Survey plates the optical counterparts are brighter than $20 \mathrm{mag}$, iii) optical spectroscopy and optical polarimetry show basically featureless polarized continuous spectra.

\section{OBSERVATIONS}

The BL Lac nature of about half of the objects finally selected was previously known in the literature. For the remaining sources various optical observing programs were carried out in order to establish their membership in the complete sample, which included :

i) spectrophotometric measurements at the Steward Observatory 90 inch and Multi Mirror telescopes in Tucson (Kuhr, Liebert, Strittmatter to be 
published), and ii) polarization measurements at the Steward Observatory 90 inch telescope (Kuhr, Schmidt, Strittmatter to be published). Subsequent direct deep deep CCD images of high quality and typical integration times of several hours were taken at the two $2.2 \mathrm{~m}$ telescopes of the Max-Planck-Institut fur Astronomie on La Silla, Chile, and on Calar Alto, Spain. The standard reduction process used included flat fielding and removal of cosmic ray events, adding and calibrating the various frames, as well as determining the point spread function and subtracting the pointlike sources from the BL Lac objects as described by Gehren et al. (1984).

\section{RESULTS}

So far only the four brightest objects of our complete sample were fully reduced and analyzed. Preliminary results indicate that the radial brightness distribution in each case is compatible with that expected for elliptical galaxies.

\section{REFERENCES}

Gehren, T., Fried, J., Wehinger, P.A. Wyckoff, S. 1984, Ap.J. 278, 11 Kuhr, H., Witzel, A. Pauliny-Toth, I.I.P., Nauber, U. 1981, Astron. Astrophys. Suppl. $\underline{45}, 367$ 